\title{
La enfermedad mental: Un problema mundial carente de tratamiento
}

\section{Objetivo}

Estimar la prevalencia, severidad y tratamiento de los desórdenes mentales, en una iniciativa de estudio de Salud mental de la OMS.

\section{Diseño}

Encuesta poblacional internacional realizada entre 2001-2003.

\section{Lugar}

Fueron llevados a cabo 15 estudios en 14 países que incluyen: América (Colombia, México y Estados Unidos), Europa (Bélgica, Francia, Alemania, Italia, España, Ucrania y Noruega), Libano, Nigeria, Japón y China. De estos países, 6 fueron clasificados por el Banco Mundial como subdesarrollados: China, Colombia, Libano, Nigeria y Ucrania.

\section{Participantes}

Todos los estudios se realizaron en casas de familia. El rango de medidas de la muestra fue sobre un total de 60.463 participantes adultos. El rango de tasa de respuesta va desde $45,9 \%$ en Francia hasta $87,7 \%$ en Colombia. Para la encuesta se utilizó el CIDI World Health Organization (WHO) Composite International Diagnostic Interview (entrevista estructurada de diagnóstico, basada en los criterios del DSM IV) que en su versión ampliada incluye preguntas detalladas sobre severidad, deterioro y tratamiento de los desórdenes mentales.

\section{Medición de resultados principales}

Los desórdenes considerados en el CIDI incluyen: Desórdenes de ansiedad, del control de los impulsos y del humor. Persistencia en adultos de déficit de atención con hiperactividad, desórdenes de conducta desafiante oposicional y por abuso de sustancias. Estos fueron clasificados como leves, moderados y graves. En un esfuer- zo por establecer categorías de severidad se interrogó sobre cuántos días en el último año fueron totalmente incapaces de llevar a cabo sus actividades habituales a causa del desorden. También se evaluó si recibieron tratamiento en el mismo período.

La prevalencia total fue muy variada desde $4,3 \%$ en Shangai a $26,4 \%$ en Estados Unidos.

Los desórdenes de ansiedad fueron los más frecuentes en la mayoría de los países (prevalencia del $2,4 \%$ a 18,2\%). Le siguen los desórdenes del humor $(0,8 \%$ a $9,6 \%)$, abuso de sustancias $(0,1 \%$ a $6,4 \%$ ) y los desórdenes del control de los impulsos $(0,0 \%$ a 6,85$)$. Las proporciones de cualquier desorden grave van de $0,4 \%$ a $7,7 \%$, los moderados de $0,5 \%$ a $9,4 \%$ y los leves de $1,8 \%$ a $9,7 \%$. La distribución de la severidad varía a través de los países, con una fuerte asociación entre la prevalencia total de algunos desórdenes y casos graves del mismo en una región.

La proporción de encuestados que recibieron tratamiento en el último año varía entre $0,8 \%$ en Nigeria a $15,3 \%$ en Estados Unidos, la cual es más alta en los casos graves $(49,7 \%-65,5 \%$ en países desarrollados vs. $14,6 \%-23,7 \%$ en subdesarrollados). Sin embargo la mayoría de la gente en tratamiento en cada país es caso leve o subumbral. El porcentaje de casos graves sin tratamiento va de $35,5 \%$ a $50,3 \%$ en países desarrollados y $76,3 \%$ a $85,4 \%$ en subdesarrollados.

\section{Conclusiones}

Existe una alta prevalencia de desórdenes mentales, a menudo asociados con severos impedimentos frecuentemente no tratados. La prevalencia total es menor en países asiáticos. En los países subdesarrollados hay mayor cantidad de pacientes sin tratamiento. Existe una importante asociación entre severidad y posibilidad de tratamiento en todos los países habiendo un alto porcentaje de tratamiento de trastornos subumbral.

\section{Comentario}

El impacto de las enfermedades mentales en la salud de las poblaciones es a veces difícil de estimar debido a la variabilidad en su diagnóstico y a las diferentes formas de medir su impacto sobre la salud ".

A su vez la implicancia que estas tienen sobre la salud de los individuos puede ser tan marcada como el de otras enfermedades crónicas quizás más estudiadas ${ }^{2}$.

En este estudio se realiza una encuesta en diferentes países utilizando un instrumento validado para el diagnóstico de algunas enfermedades mentales por personas sin un entrenamiento específico en el área de la salud mental ${ }^{3}$ lo que en cierta forma trata de unificar los criterios diagnósticos de las mismas.

Una importante limitación del estudio es la amplia variación en la tasa de respuesta de la encuesta, que en algunos casos estuvo por debajo del estándar. La esquizofrenia y otras psicosis no afectivas fueron excluidas. Sin embargo, quedaron incluidas reuniendo criterios de ansiedad, abuso de sustancias, etc., que sí valora el CIDI. Los países con la menor prevalencia estimada de desórdenes tienen la mayor proporción en tratamiento de casos subumbral, lo que "podría reflejar una subestimación de los desórdenes".

Es importante destacar que fueron excluidos los pacientes hospitalizados, lo que subestima la proporción de casos graves que reciben tratamiento. La existencia de muchos pacientes con desórdenes subumbral que reciben tratamiento mientras que muchos otros con desórdenes graves no lo reciben demuestra que no es simplemente un problema de recursos limitados, aunque este aspecto pueda estar involucrado. La complejidad en la redistribución de los recursos se da en la falta de reglas explícitas, la percepción distorsionada de las necesidades de tratamiento y las diferencias en el acceso a la cobertura social. No tratar casos leves, especialmente en gente joven, implica más adelante tener más casos graves, aunque se carece de estudios sobre características predictoras de riesgo. Es necesario poner el foco en el desarrollo de programas de prevención secundaria para el tratamiento de casos leves, y que esto guíe la racionalización en la distribución de los recursos.

Conclusiones del comentador:El porcentaje de pacientes con desórdenes mentales que no recibe tratamiento es alarmante. El desconocimiento de la necesidad de tratamiento es la clave, conjuntamente con la falta de racionalización en la distribución de recursos. En el área de salud mental, la prevención es la palabra ausente.

\section{Dra. Gabriela La Monica. Psiquiatra [ La Plata. Buenos Aires.]}

La Monica G. La enfermedad mental: Un problema mundial carente de tratamiento..Evid. actuel. práct. ambul 2004;7:141.Comentado de: Prevalence, severity, and unmet need for treatment of Mental Disorders in the World Health Organization World Mental Health Surveys. Ronald C. Kessler, T. Bedirhan Ustun. JAMA 2004. June 2, Vol. 291, №21. pag. 2581 al 2588.

\section{Referencias}

1.Murray CJL, Lopez AD. The Global Burden of Disease: A Comprehensive Assessment of Mortality and Disability From Diseases, Injuries and Risk Factors in 1990 and Projected to 2020. Cambridge, Mass: Harvard University Press; 1996

2.Ormel J, VonKorff M, Ustun TB, Pini S, Korten A, Oldehinkel T. Common mental disorders and disability across cultures: results from the WHO Collaborative Study on Psychological Problems in General Health Care. JAMA. 1994; 272:1741-1748.

3.Robins LN, Wing J, Wittchen H-U, et al. The Composite International Diagnostic Interview: an epidemiologic instrument suitable for use in conjunction with different diagnostic systems 